\title{
Ikonographie, Ikonizität und Ikonizismus: Drei Begriffe und ihre Bedeutung für die Phraseologieforschung
}

\author{
Ken Farø (Kopenhagen)
}

\begin{abstract}
Three key concepts in the research of idioms are discussed: iconography, iconicity, and iconicism. These concepts all have a role to play in phraseology (and beyond), although in quite different ways: Iconography is the mental picture that may be created on the basis of the literal reading of an idiom; it is thus a genuine semiotic concept and not a matter of free associations. However, its functional relevance in discourse should always be questioned. Iconicity in phraseology is the phenomenon that many idioms seem to reflect their semantic content through their form. Although this seems intuitively correct, idiom iconicity is quite relative and always overruled by the arbitrariness of the idiom sign - an arbitrariness which is not relative, but in fact fundamental. Iconicity is therefore often an obstacle to more thorough empirical investigations and cross-linguistic contexts. Iconicism is the tendency found in research and applied contexts that more attention is paid to iconography than to the idioms' rules of use. Iconicism is among other things responsible for textual non-equivalence and nonnative style in translations, and it is a "natural", but nevertheless problematic consequence of the iconography and iconicity of idioms.
\end{abstract}

\section{$1 \quad$ Einleitung}

In diesem Beitrag werden drei teilweise etymologisch verwandte Begriffe diskutiert, die wenn auch auf verschiedene Weise - eine wichtige Rolle (nicht nur) für die Phraseologieforschung spielen können. Ihre Anwendung setzt jedoch voraus, dass sie genau definiert und in der Praxis sauber getrennt werden. Es handelt sich um eine Begriffs-Triade, die bisher nicht im Zusammenhang diskutiert wurde, obwohl sie nicht zuletzt in einer solchen Gesamtperspektive von theoretischem Nutzen sein könnte. Und zwar geht es um die das Element Ikon- enthaltenden Termini Ikonographie, Ikonizität und Ikonizismus, von denen vor allem der letztere besondere Beachtung verdient, weil er in allen Bezügen neu ist. Obwohl es sich um keine empirische, sondern um eine begrifflich-theoretische Untersuchung handelt, basiert die Diskussion auf empirischen Analysen, in erster Linie aus Farø (2006).

\section{Theoretischer Hintergrund}

Bevor ich die eigentliche Diskussion beginne, soll ihre theoretische Basis erörtert werden. Zwei wichtige Grundlagen sind zu besprechen, nämlich die Arbitraritätstheorie und der 
allgemeine linguistische Funktionalismus. Diese sind entscheidend für eine Idiom-Theorie, die auch dem Sprachvergleich und angewandten Zusammenhängen gerecht sein und nicht nur Post-hoc-Erklärungen liefern will, d.h. solche, die bloß intuitiv richtig erscheinen. Die Anwendungsperspektive ist für die Theorien in hohem Maße verpflichtend und stellt deshalb eine große Herausforderung dar.

\subsection{Arbitraritätstheorie}

Ein arbitraritätstheoretischer Zugang zur Phraseologie geht davon aus, dass sich Idiome semiotisch nicht von anderen lexikalisierten Sprachzeichen unterscheiden und somit arbiträre Zeichen sind (cf. Farø 2006 und im Druck). "Arbitrarität" ist dabei strikt linguistischterminologisch zu verstehen, und nicht, wie er oft (miss-)verstanden wird, gemeinsprachlich: im Sinne von 'Willkür' oder 'Nicht-Motivierbarkeit'/'Opazität' ${ }^{1}$. Arbitrarität im linguistischen Sinn impliziert u. a.:

(1) Systemgebundenheit des Sprachzeichens;

(2) keine prinzipielle Notwendigkeit ("Natürlichkeit") der Beziehung von Ausdruck und Inhalt;

(3) Nicht-Ableitbarkeit des Inhalts aus dem Ausdruck;

(4) Nicht-Herleitbarkeit von Äquivalenz aufgrund von Ausdruckskonvergenz zwischen L1und L2-Idiomen (= "interlinguale Arbitrarität").

Auf diese Aspekte soll im Folgenden kurz eingegangen werden.

Ad (1): Idiome sind als Zeichen nur als Elemente eines gegebenen Sprachsystems adäquat zu verstehen. Ohne dieses System, das ihnen überhaupt erst eine Funktion zuerteilt, sind sie keine Idiome. Das System selbst ist eine Abstraktion, die auf den kummulativen Zeichenverwendungen der daran teilnehmenden Sprecher und den daraus entstehenden Gebrauchsregeln basiert. Der "Inhalt" - an sich eine Metapher - des Idioms ist nicht nur als die Semantik i.e.S. zu verstehen, sondern als seine gesamten Gebrauchsregeln, die der idiomkompetente Sprecher beherrscht, d. h. auch Pragmatik, Syntax etc. Idiome außerhalb des Systems betrachten zu wollen ist problematisch, weil der "Inhalt" sich nur relativ zum System konstituiert; ohne System kein "Inhalt".

Ad (2): Für die Sprecher einer Sprache scheinen die Verbindungen der Ausdrucks- und Inhaltsseite "ihrer" Idiome "natürlich" und notwendig zu sein. Dass es prinzipiell anders sein könnte, und dass es in der Tat oft auch anders ist, fällt ihnen gar nicht ein. So ist es für einen dänischen Sprecher merkwürdig, dass jm die Stange halten auf (Bundes-)Deutsch 'jm helfen' heißt, wo doch holde ngn stangen auf Dänisch das Gegenteil bedeutet. Genauso ist es merkwürdig, dass am grünen Tisch 'theoretisch, nicht-empirisch' u.ä. bedeutet, wenn man mit dem Glauben aufgewachsen ist, dass dänisch ved det grønne bord die 'Examenssituation' bezeichnet. Auf diese Tatsachen haben - wenn auch in einem nicht-phraseologischen,

\footnotetext{
1 Vielleicht sind Saussures (1916) Beispiele (arbre, boeuf, soeur) und sein Abschnitt über Motivation Teilursachen für dieses Missverständnis (cf. auch Holdcroft 1991).
} 
allgemein linguistischen Kontext - u. a. Whitney (1876) und Saussure (1916) hingewiesen und sie gehören zu den Hauptargumenten für die lexikalische Arbitrarität. Ein zusätzliches Argument ist die prinzipielle diachrone Wandelfähigkeit sowohl des Ausdrucks als auch des Inhalts.

Ad (3): Ein weiteres Element kann als die "intrasemiotische Nicht-Ableitbarkeit" bezeichnet werden, auf die z. B. Bolinger (1968: 15) und Rapoport (1975: 6) hingewiesen haben. Denn die Bedeutung eines Idioms ist prinzipiell nicht aus seiner Form herleitbar, sondern nur aus seinem Gebrauch. Mit phraseologischen Ratestrategien (cf. Hallsteinsdóttir 2001: 210f.) kann man manchmal Erfolg haben, der Erfolg muss sich aber am Gebrauch und dem System messen lassen. Es wäre deshalb falsch, solche singulären Erfolgserlebnisse als Aufhebung der Arbitrarität zu bewerten. Diese Art Motivierbarkeit von Idiomen basiert auf individuellen Hypothesen, deren Erfolg keineswegs garantiert ist. Hier geht es nicht um solche individuellpsychologische Methodik, sondern um die systematische Nicht-Ableitbarkeit der Bedeutung aus der Form eines Idioms.

Ad (4): Die Arbitrarität von Idiomen impliziert auch, dass Äquivalenzfragen nicht anhand der Ausdrucksseite von interlingualen Idiompaaren entschieden werden können. Wenn man den Verdacht hat, zwei Idiome aus verschiedenen Sprachen "heißen dasselbe", dann kann diese Hypothese nur durch Studien ihrer Gebrauchskontexte entschieden werden. Die Ausdrucksseite an sich liefert keine Garantie für die Richtigkeit der Hypothese.

Die Arbitrarität, gerade auch von anscheinend stark "motivierten" Sprachzeichen wie vielen Idiomen, ist ein so grundlegendes Prinzip der Sprache ${ }^{2}$, dass man es leicht ignoriert oder missversteht. Es ist aber nicht zuletzt deshalb theoretisch so wichtig, weil es auch methodologische Konsequenzen hat.

\section{2 (Genuiner) Funktionalismus}

Ein Zugang zur Idiomatik, der funktionalistisch ist, ist eine weitere Grundlage für das Verständnis der drei zu besprechenden Begriffe. In seinem hier relevanten allgemeinen Sinn ist der linguistische Funktionalismus als ein 'Anti-Essentialismus' zu verstehen, dass also aus der Existenz von etwas nicht unbedingt eine bestimmte Essenz desselben folgt. Damit ist im phraseologischen Zusammenhang gemeint, dass vom Auftreten eines Idioms weder automatisch auf (1) seine intendierte Autor-Funktion im Text noch (2) auf seine adäquate Handhabung in interlingual-angewandten Zusammenhängen (v. a. Übersetzung und bilingualen Wörterbüchern) geschlossen werden kann. Dies ist nämlich beides rein kontextuell bestimmt und hängt nicht 1:1 vom Idiom selbst ab, sondern von den Funktionen, die es im sprachlichen Zusammenhang hat, bzw. denen im Kontext Relevanz zugeschrieben wird. Letzteres gilt z. B. für die Lexikographie. Der funktionalistische Zugang zur Idiomatik geht von den konkreten, aktuellen Funktionen aus. Er nimmt nicht an, dass Idiome an sich besondere Funktionen ausüben, weil sie Idiome sind, oder dass sie z. B. nach festen Regeln

\footnotetext{
2 Saussure (1916) nannte sie "das erste Prinzip", für Hockett (1958: 569-580) gehörte sie zu den sieben "key properties" natürlicher Sprachen.
} 
übersetzt werden müssen, wie es die translatologische Literatur häufig vermittelt. Im Folgenden soll anhand von wenigen Beispielen gezeigt werden, was dieser Funktionalismus impliziert.

Ad (1) Idiome in Texten: Wenn man ein Beispiel wie dieses liest:

(1.) Er schleppte so einen Wälzer herum[...], um immer[...] seinen Senf dazugeben zu können

(Schulze 1998: 16)

geht man bei einem funktionalistischen Zugang nicht automatisch davon aus, dass dahinter eine besondere Intention des Autors steckt, die nicht genau so gut von einer anderen Formulierung hätte abgedeckt werden können. Die Tatsache, dass das verwendete Sprachzeichen ein Idiom ist, heißt nicht, dass der Autor damit eine bewusste Strategie verfolgt hat, dass er sich unbedingt "idiomatisch" oder "bildlich" hat ausdrücken wollen (cf. Häcki Buhofer 1997: 223). Denn damit misst man der Phraseologie zuviel an Intentionalität bei, die der realen Kommunikation nicht gerecht wird. Wer bei sich selbst versucht hat, systematisch-introspektiv nachzuprüfen, wie und wann er/sie Idiome verwendet, wird auch festgestellt haben, dass Idiome kaum bewusst-strategisch als Idiome verwendet werden, sondern eher weil ihre Gebrauchsregeln zum Kontext passen oder weil sie aus mnemotechnischen Gründen für idiomkompetente Sprecher schnell greifbar sind. Der idiomatische Funktionalismus lehnt es ab, dem Gebrauch von Idiomen eine kommunikative Funktion beizumessen, die die von Einzelwörtern prinzipiell transzendiert. Idiome sind in diesem Sinn normallexikalische Einheiten und haben keine Funktionen, die nicht auch grundsätzlich bei Monolexemen vorkommen. Gewisse Tendenzen zur Informalität und pragmatischer Markiertheit ändern nichts daran, denn diese Eigenschaften finden sich auch bei Einzelwörtern. Die Kategorie ist also als solche kommunikativ betrachtet nichts Besonderes, auch wenn ihre Entstehung mit einem bestimmten kommunikativen Bedürfnis eines Sprechers verknüpft werden kann. Aber gerade in dieser Phase waren sie noch keine Idiome, denn dazu ist erst eine Lexikalisierung nötig.

Ad (2) Interlingual-angewandte Zusammenhänge: Der allgemeine Idiomfunktionalismus bewahrt auch in interlingual-angewandten Zusammenhängen ihre Gültigkeit, sein Nutzen wird hier vielleicht erst recht deutlich. Denn wenn die Funktion von Idiomen in der Sprache nicht von vornherein gegeben ist - sozusagen als Kategorialfunktion -, sondern eine Sache der (zugegeben nicht immer einfachen) Analyse vor Ort ist, dann können auch keine allgemein verbindlichen, kontextlosen Regeln formuliert werden, wie Idiome interlingual gehandhabt werden sollten -, abgesehen von der Regel, dass man sie funktional adäquat behandeln sollte. Die Versuche der bisherigen Übersetzungshandbücher, absolute Anweisungen zur Idiomübersetzung zu geben (z. B. Ingo 1990: 209ff.), überzeugen nicht, denn sie basieren offensichtlich nicht auf empirischen Idiomgebrauchsstudien, sondern auf der Vorstellung einer besonderen "Farbigkeit" von Idiomen (vgl. Fußnote 8). In der Praxis gehen Übersetzer denn auch vielfach, wenn auch nicht konsequent funktionalistisch mit Idiomen um, vgl:: 
(2.) [hun] tog sin hund under armen og fløj af sted med en forfatter i maven $\rightarrow$ [sie] nahm ihren Hund unter den Arm und flog mit ihren Schriftstellerambitionen davon

(Brøgger 1997/2001: 274/277)

Das dänische Idiom kann als "[PERSON, die man gern sein möchte] im Magen/Bauch haben" literalisiert werden. Dieser Inhalt wurde vom Übersetzer funktional adäquat übersetzt, denn die Verwendung des Idioms repräsentiert vermutlich keine besonderen bzw. nachvollziehbaren Intentionen des Autors.

Der Idiomfunktionalismus im Übersetzungszusammenhang entspricht auch einem lexikographischen Funktionalismus. In bilingualen Wörterbüchern geht es zumindest nicht nur darum, Idiome des L2 zu finden, die mit den L2-Idiomen "funktional äquivalent"3 sind, im Sinne davon, dass sie "gleich verwendet" werden, denn dies ist grundsätzlich eine lexikologische Perspektive auf die Lexikographie (cf. Farø 2004 und Almind et al. in diesem Heft). Vielmehr geht es in der Lexikographie darum, lexikographische Funktionen wie z. B. Rezeption, Produktion, Wissen oder Übersetzung für den Benutzer zu erfüllen (cf. u. a. Tarp 1995 und Bergenholtz/Tarp 2002). Die Art der Erfüllung dieser Funktionen im Wörterbuch ist nicht von vornherein gegeben, nicht einmal ob sie überhaupt sämtlich abgedeckt werden sollen. Dies kommt völlig auf das Wörterbuchkonzept an, das mit seinen intendierten Funktionen gerade deshalb transparent umgehen muss. Die lexikographische Funktion Rezeption könnte z. B. ein anderes Herangehen an ein L1-Idiom nahelegen als Produktion oder Wissen usw. (cf. Farø 2006).

Wichtig am Idiomfunktionalismus ist also, dass das Vorhandensein eines Idioms in einem Text oder als Problem in einem angewandten Zusammenhang immer eine realistische Analyse seiner aktuellen Funktion oder der relevanten Merkmale des Idioms im aktuellen Zusammenhang nötig macht. Idiomfunktionalismus ist Relevanzanalyse anhand des Kontexts. Idiome treten in verschiedenen Kontexten auf, die jeweils verschiedene potenzielle Aspekte des Idioms relevant machen, während andere bedeutungslos bleiben. Diese Analyse muss ad hoc vorgenommen werden und kann nicht kontextlos oder en bloc von Idiomen an sich, ja nicht einmal von einem einzelnen Idiom gemacht werden.

\section{$3 \quad$ Ikonographie}

Der erste der drei zu diskutierenden Begriffe, die Ikonographie ${ }^{4}$, ist das Ergebnis des Versuchs, die gemeinsprachliche "Bildlichkeit" (cf. Burger 1989 sowie Burger im Druck),

3 Dobrovol'skijs (z. B. 1999a, 1999b, 2000, 2002) so genannte "funktionale Idiomäquivalenz" ist richtungsweisend, weil differenzierend, er ist m.E. aber nicht genuin funktionalistisch und somit für die Phraseologieforschung nicht flexibel genug. Denn ein genuiner Idiomfunktionalismus geht nicht generell davon aus, dass "ein gutes L2-Äquivalent des L1-Idioms [...] kein Idiom zu sein" brauche (Dobrovol'skij 1999a: 114), vielmehr muss darüber der Zusammenhang entscheiden. Der "funktionale Idiomäquivalenzbegriff" Dobrovol'skijs ist auch deswegen nicht genuin funktionalistisch, weil er die Kontexte Übersetzung und Lexikographie im Grunde genommen lexikologisch betrachtet und nicht aufgrund eigener funktionalistischtranslatorischer bzw. -lexikographischer Theorien (s. u.).

${ }^{4}$ Er stammt aus der Kunstgeschichte (cf. Panofsky 1978). 
über deren Auslegung in der Phraseologieforschung keine Einigkeit besteht (Krohn 1994: 75), durch einen internationalistischen Fachterminus auszutauschen und ihn bei dieser Gelegenheit verbindlich zu definieren. Die Vorteile von Internationalismen sollten nicht unterschätzt werden. Sie

(1) erleichtern die internationale Fachkommunikation,

(2) sind meistens morphologisch hochflexibel, und

(3) verringern die Gefahr subjektiver Interpretationen des Terminus.

Das Vorkommen eigenwilliger Interpretationen von Termini ist ein bekanntes Phänomen. Ein Beispiel dafür ist die Einstufung des Syntagmas sich ergeben ('kapitulieren') als ein "Idiom" von einer meiner Studenten. Als Begründung nannte sie die Mehrwortstruktur und eine angebliche "Bildlichkeit". Es zeigte sich, dass dieser Interpretation folgende Überlegungen zugrundelagen: "Man sieht die Waffen auf dem Boden, die hoch gehaltenen Hände und die weiße Fahne". Hier wird offensichtlich eine Art "Script" konstruiert, das "bildhaft" ist, trotzdem wird damit noch keine Ikonographie beschrieben. Denn diese verstehe ich ausschließlich als eine auf der literalen ("wörtlichen") Lesart (cf. Burger 2003) basierende Interpretation, und eine solche besitzt sich ergeben im Gegensatz etwa zum semantisch transformierten Idiom jn über den Tisch ziehen nicht. Die Ikonographie ist also nicht einfach eine "Bildlichkeit" im Sinne Häcki Buhofers (1989: 166):

"eine Eigenschaft der Sprache[...], bei der es darum geht, wie gut man sich dazu visuell etwas vorstellen kann",

d. h. ein rein psychologisches Merkmal, sondern die Ikonographie ist eine semiotischpsychologische Eigenschaft von Sprachzeichen. Übrigens teilen sich Idiome i. e. S. - die man geradezu anhand dieses Merkmals definieren kann (cf. Farø 2006) - die Ikonographie mit vielen Monolexemen, etwa Komposita (z. B. Säbelgerassel) und Simplizia (z. B. Bulle, abschätzig-salopp für 'Polizist').

Der Begriff "Ikonographie" ist fester umrissen und liefert sich weniger subjektiven Interpretationen aus als der Begriff "Bild(lichkeit)", der viele Funktionen hat und außerdem ein gemeinsprachlicher Begriff ohne terminologische Verpflichtung ist.

\section{$4 \quad$ Ikonizität}

Die Frage, ob Idiome ikonische Zeichen sind, ist noch nicht zufriedenstellend beantwortet worden. Einfach zu sagen, dass Idiome "bildhaft" und somit ikonisch sind, ergäbe keine theoretisch akzeptable Antwort, denn Ikonizität ist, trotz des Ikon-Elements, etwas anderes und mehr als bloß "Bildhaftigkeit". Während hier die Ikonographie als eine absolute Eigenschaft von Idiomen (i.e.S.) verstanden wird, ist die Ikonizität eindeutig als eine relationale Eigenschaft auzufassen, nämlich als eine nachvollziehbare Beziehung von Ausdruck und Inhalt. Die semiotisch-phraseologische Handhabung der Ikonizität hängt von mehreren Faktoren ab, u. a. davon, wie Ikonizität überhaupt verstanden wird, und von der Granularität der Beschreibung. 
Ikonizität wird meistens als ein "Abbildungsverhältnis" verstanden, als eine Art "form miming meaning" (Nänny/Fischer 1999: Titel). Ikonische Zeichen sind

"those whose relation to their objects is a mere community in some quality, and these representations may be termed Likenesses"

(Lyons 1977: 102)

Ich betrachte somit Ikonizität als einen Spezialfall von Motivation (die man terminologisch sauberer als "Motivierbarkeit" bezeichnen sollte, vgl. Farø 2006). Dass bestimmte Phraseologismen im gewissen Sinn ihren Inhalt "abbilden", d. h. für diejenigen semantisch transparent sind, die ihre Bedeutung schon kennen (Herbermann 1981: 347), wird deutlich, wenn man beispielhaft die Idiome (a) unters Messer kommen, (b) gesiebte Luft atmen, und (c) jm grünes Licht geben betrachtet. Diese Idiome bilden durchaus etwas ab, das mit ihrem Inhalt zusammenhängt: In (a) wird das Messer als chirurgisches Kerninstrument metonymisch für die ganze Operation verwendet; in (b) wird, metonymisch-metaphorisch gefiltert, der Gefängnisaufenthalt mit Fokus auf das übliche Gitter vorm Fenster (metonymisch) betrachtet, das wiederum metaphorisch als Luft-Sieb gesehen wird; in (c) ist die Abbildung rein metaphorischer Art, indem das Erlaubnis-Geben mit der Erlaubnis zum Fahren an einer grünen Ampel verglichen wird. Obwohl stark perspektivisch, kann in allen Fällen von einer Art Abbildung des Inhalts durch den Ausdruck gesprochen werden. In diesem Sinn sind die Idiome auch "ikonisch". Es kann aber auch nur von einer sehr grob granulierten ${ }^{5}$ Abbildung gesprochen werden. Es wird nämlich bloß eine kleine Menge der Inhaltseigenschaften des Idioms abgebildet. Somit kann keineswegs davon ausgegangen werden, dass "form and meaning are one" (Fischer 1999: 124), was nach Fischer (ebd.) bei Ikonizität zumindest "in its ideal form" der Fall sei.

Die Ikonizität von sehr vielen Idiomen ist eine retrospektiv festgestellte Widerspiegelung des Inhalts im Ausdruck (= "Transparenz"). Die Feststellung von Ikonizität ist aber theoretisch eine recht schwache These, denn:

(1) Der Inhalt ikonischer Idiome kann prinzipiell nicht prospektiv bestimmt werden, dazu ist eine genaue Analyse des Sprachgebrauchs nötig. Außerdem ist wichtig, dass die Ikonizität stark subjektiv sein kann und in jedem Fall perspektivisch ist: Was für einen Sprecher eindeutig ikonisch ist, kann für einen anderen Sprecher ohne Ikonizität sein. Interlinguale Vergleiche "offenbar" ikonischer Zeichen wie der Onomatopoetika (z. B. peng, gäköl, curlew) zeigen, dass die Ikonizität häufig verloren geht, wenn sie Sprachgrenzen überschreitet.

(2) Die Ikonizität kann unmöglich eine Beziehung zwischen allen Ausdrucks- und Inhaltselementen repräsentieren. Ein erweitertes semiotisches Verständnis von "Inhalt", das

\footnotetext{
5 In Farø (2006) wird argumentiert, dass dieser Aspekt in der Phraseologieforschung zu wenig Aufmerksamkeit erhält. Viele Konklusionen sind davon abhängig, wie fein granuliert die Analyse ist, d.h. wie hoch die Toleranzschwelle gegenüber Abweichungen bei Vergleichen angesetzt wird. V. a. in der kontrastiven Liguistik fehlen standardisierte Maßstäbe für die Genauigkeit der Analyse.
} 
z. B. auch die Pragmatik und Syntax sowie weitere zeichenrelevante Merkmale dazurechnet ${ }^{6}$, ist mit der gängigen Ikonizitätstheorie nur schwer zu vereinbaren.

(3) Die Granularität von Analysen, die sich mit Ikonizitätsfragen beschäftigen, ist in der Regel sehr grob; was nicht an sich gegen sie spricht. Es berechtigt aber zur Frage nach der systematisch-kommunikativen Relevanz der Ikonizität.

Diese 3 Einwände sind auch ein Teil der Erklärung dafür, dass die prinzipielle Arbitrarität und die eventuelle Ikonizität von Idiomen keinen Widerspruch darstellen: Die Arbitrarität reguliert alle Details des Zeichengebrauchs und ist das zentrale kommunikative Prinzip. Und da sie von einer eventuellen Ikonizität nicht affiziiert wird, ist auch kein begrifflicher Konflikt vorhanden. Die Ikonizität ist im hohen Grade relativ, weil sie graduierbar ist (cf. Dobrovol'skij/Piirainen 2005: 71). Die Arbitrarität dagegen ist ein absolutes Prinzip. Die Ikonizität ist für die Kommunikation meistens irrelevant, wie der Vergleich von äquivalenten ikonischen und nicht-ikonischen Zeichen verschiedener Sprachen zeigt.

Die Ikonizität und die Ikonographie bilden die formal-semiotische Grundlage für die Existenz des Phänomens Ikonizismus, das im nächsten Abschnitt erklärt wird.

\section{$5 \quad$ Ikonizismus}

\subsection{Zum Begriff}

Der Begriff Ikonizismus ist das jüngste Mitglied der phraseologisch relevanten Ikon-Familie und soll hier deshalb eingehend besprochen werden. Er wurde in Farø (2004) als eine Reaktion auf anti-arbitraritätstheoretische und anti-funktionalistische Zugänge zur Idiomatik in der Forschungsdiskussion und in angewandten Zusammenhängen eingeführt. Es handelt sich beim Ikonizismus um eine interpretatorische Perspektive, und nicht um eine Eigenschaft von Idiomen. Der Begriff Ikonizismus kann als eine Folge der Ikonographie und Ikonizität von Idiomen gesehen werden. Seine Funktion ist v. a. die Wirkung als Korrektiv, nicht zuletzt in der interlingualen Idiomhandhabung wie sie in Übersetzungen und bilingualer Lexikographie deutlich wird, in der Idiome praktische Probleme verursachen. Der Ikonizismus ist ein Komplex, der aus den folgenden Elementen besteht:

(1) In semantischen Analysen von Idiomen wird der Ikonographie das Hauptgewicht beigemessen, nicht den abstrakten Gebrauchsregeln des Idioms.

(2) Die kommunikative Relevanz der Ikonizität von Idiomen wird häufig überschätzt, v. a. wenn sich die Inhaltsanalyse in einer der Ikonographie entsprechenden und motivierenden Beschreibung erschöpft.

(3) In interlingualen Zusammenhängen wird eine kommunikative Äquivalenz von Übereinstimmungen auf der Ausdrucksebene abhängig gemacht. Dies bedeutet, dass ein Mehr

\footnotetext{
${ }^{6}$ Denn es gibt keine theoretisch gültigen Argumente, die für eine Priorität der Semantik in diesem Zusammenhang sprechen.
} 
an Ausdruckskonvergenz mit einem Mehr an Äquivalenz gleichgesetzt wird, was nicht der Fall sein muss.

Das Ziel der folgenden Diskussion ist es zu zeigen, dass ein praktizierter Ikonizismus nur eine sehr begrenzte theoretische Perspektive bietet und außerdem mit wesentlichen Problemen verbunden ist.

\subsection{An allen Ecken und Enden}

Dobrovol'skij (2001) untersucht das ikonische Idiom an allen Ecken und Enden unter der Überschrift der "Motivation", die als ein Hyperonym für "Ikonizität" gesehen werden kann. Er stellt fest, dass sich das Idiom "- ganz im Einklang mit seinen bildlichen Bedeutungskomponenten - nur auf bebaute und bewohnte Räume beziehen kann" (op. cit.: 90). Es ist diese Analyse des "Einklangs" von Ausdruck und Inhalt, die dazu berechtigt von einem Fall von Ikonizität zu sprechen, obwohl das Wort bei Dobrovol'skij selbst nicht auftaucht. Dobrovol'skij äußert sich hier, von der ikonographischen Ausdrucksseite ausgehend, über mögliche und unmögliche Realisierungen des Idioms. Solche Thesen sind falsifizierbar und somit i.e. S. wissenschaftlich (cf. Popper 1935), also auch systematisch erfassbar.

Im Zeitalter von Textkorpora und des Internets ist das Verfahren der linguistischen Falsifizierung einfacher geworden. Es ist richtig, wie Dobrovol'skij feststellt, dass "bebaute und bewohnte Räume" zu den Realisierungskontexten von an allen Ecken und Enden gehört, vgl.z. B.:

(3.) Stil, Komfort und Gastfreundschaft an allen Ecken und Enden. Komfortabel und gemütlich:

27 Doppelzimmer, 2 Einzelzimmer und 2 Studios Zimmer

(www.evviva.de)

(4.) An allen Ecken und Enden gab es Leute, die auf ihren iBook und Powerbooks rumtippten, im Flur zwischen den Vortragssälen standen mehrere iMacs und die Videotechnik wurde mit eMacs bewerkstelligt

(www.dabo.de)

(5.) Einen Problempunkt hat der Verein allerdings: das Sportheim, das an allen Ecken und Enden saniert werden muss

(www.gea.de)

In (3.) ist von einem Hotel die Rede, in (4.) von einem Konferenzort, d. h. auch einem Gebäude irgendeiner Art, in (5.) ebenso. Das ist vielleicht der ursprüngliche Realisierungskontext des Idioms. Er ist aber keineswegs der einzig mögliche, nicht einmal unbedingt der häufigste, wie folgende, bei weitem nicht untypische Realisierungen des Idioms zeigen:

(6.) Die deutsche Sprache zerfällt an allen Ecken und Enden

(www.vds-ev.de) 
(7.) Das Bike klappert an allen Ecken und Enden

(www.1200bandit.de)

(8.) Sie hat ein faszinierend schlechtes Buch hinterlassen, das an allen Ecken und Enden holpert und klappert

(www.literaturkritik.de)

(9.) Es fehlt an allen Ecken und Enden und wie man hört, mussten sogar mancherorts Rechnungen aus dem Vorjahr aus dem heurigen Budget bezahlt werden

(www.kritikus.at)

(10.) die Staatsverschuldung steigt weiter, aber an allen Ecken und Enden des einstigen sozialen Netzes wird geschnitten und gekürzt

(www.heise.de)

In diesen sowie in vielen anderen Belegen kommt das Idiom in recht unterschiedlichen Realisierungskontexten vor, z. B. im Zusammenhang mit Sprache, Fahrrädern, Büchern und Budgets. Die These Dobrovol'skijs, das Idiom könne nur auf "bebaute und bewohnte Räume" bezogen werden, lässt sich anhand von Korpusstudien leicht falsifizieren.

Ikonizistisch an diesem Zugang ist die Vorstellung, dass die Ikonographie bestimmte Gebrauchsrealisierungen prinzipiell restringiere, dass keine arbiträre Beziehung zwischen dem Ausdruck und dem Inhalt bestehe. Das ist nicht nur eine falsche, sondern auch eine methodologisch gefährliche Vorstellung. Nicht die Ikonographie restringiert den Gebrauch, sondern der systematische Gebrauch der Sprecher restringiert den individuellen Gebrauch des Idioms.

\subsection{Eulen nach Athen tragen vs. gå over åen efter vand}

Ein weiterer Fall von Ikonizismus stammt aus der bilingualen Metalexikographie. In einer Diskussion ${ }^{7}$ darüber, welches Idiom "das richtige" deutsche Wörterbuchäquivalent des dänischen Idioms gå over åen efter vand sei, äußerte sich der Lexikograph Axelsen (1992: 1f.) folgendermaßen (meine Übersetzung):

Gå over åen efter vand heißt 'sich überflüssige Mühe machen um etwas zu erreichen': das Wasser kann ja genauso gut auf der Seite des Wasserlaufs geholt werden, auf der man sich gerade befindet (aber Wasser bekommt man trotzdem), während Eulen nach Athen tragen 'etwas vollständig Nutz- und Sinnloses unternehmen' heißt, weil sie ja in Athen überhaupt keine Eulen brauchen; der deutsche Ausdruck ist deshalb nicht verwendbar.

Bemerkenswert ist dabei das unbeschwerte Hinübergleiten Axelsens von der Inhalts- auf die Ausdrucksebene. Die Bedeutung ist hier offenbar eine direkte Funktion der Komposition und der Metapher; aus der Ikonographie lassen sich dann verschiedene Schlussfolgerungen ziehen. Nämlich sowohl im Rahmen der Ikonographie selbst, als auch von der Ikonographie auf die Bedeutung, und von dort wiederum auf die Frage nach interlingualer Äquivalenz.

\footnotetext{
${ }^{7}$ Anlass war das Erscheinen eines neuen dänisch-deutschen Wörterbuchs. Für eine genauere Besprechung cf. Farø $(2004,2006)$.
} 
Axelsen kann auf dieser Grundlage mit großer Sicherheit behaupten, dass Eulen nach Athen tragen kein lexikographisches Äquivalent von gå over åen efter vand sein könne, was wiederum eine neue (nämlich: disziplinäre) Ebenentranszendierung darstellt.

Diese ikonizistische Methodik ist sowohl arbitraritätstheoretisch als auch funktionalistisch gesehen unhaltbar.

\subsection{Beispiele aus Übersetzungen}

Ein weiterer Makrokontext, in dem Idiome zu ikonizistischen Interpretationen einladen, ist die Übersetzungspraxis, in der die Äquivalenzvorstellungen von Übersetzern von der Ikonographie der Idiome stark beeinflusst werden. Ausdruck einer ikonizistischen Idiomhandhabung sind z. B. diese Beispiele aus Übersetzungen aus dem Dänisch-Deutschen Idiomübersetzungskorpus (DDIK) in Farø (2006: Anhang):

(11.) "In der Fahrschule habe ich Blut und Wasser geschwitzt". "Warum? Sie sind eine gute Fahrerin" - (")Det kostede blod, sved og tårer på køreskolen." "Hvordan kan det være? De er en god bilist"

(Hein 2001/2002: 73/66)

Was dieses Beispiel ikonizistisch macht, ist die Tatsache, dass die Idiome Blut und Wasser schwitzen ('in einem Zustand großer Angst oder Aufregung sein') und ngt koster blod, sved og tårer ("etw kostet Blut, Schweiß und Tränen": 'etw fordert jm große Anstrengung und Aufopferung ab') keineswegs die gleiche Bedeutung haben. Damit ändert der Übersetzer den Textsinn - dem das Hauptaugenmerk des Übersetzens gelten sollte -, weil auf die IdiomKomponenten mehr Wert gelegt wird als auf den konventionellen Inhalt der beiden Idiome. Eine wichtige Ursache ist die partielle Konvergenz der Ikonographie (Blut und schwitzen bzw. blod und sved). Der Ikonizisimus kann also zu fehlender Textäquivalenz führen. Das ist auch im folgenden Beispiel der Fall:

(12.) Min far har bevæget sig gennem det meste af sit liv med hovedet under armen - Den größten Teil seines Lebens hat mein Vater den Kopf unter dem Arm getragen

(Høeg 1992/1996: 39/43)

Fräulein Smillas Vater, der im dänischen Original ein etwas gedankenloser Typ ist, wird durch die ikonizistische Übersetzung zu einem Mann, der sein Leben lang sehr krank, ja dem Tode nahe gewesen ist. Das ist kaum als ein subtiler Textunterschied zu bezeichnen.

Nicht nur Äquivalenzprobleme sind die Folge von Ikonizismus in Übersetzungen, sondern auch die Natürlichkeit des Zieltextes leidet nicht selten unter dieser "Bilderorientierung". So z. B. in:

(13.) "das ist nichts Ungewöhnliches, daß Ihnen der Kreislauf den Dienst verweigert. Das ist doch unser tägliches Brot" - "det er slet ikke usadvanligt, at kredslфbet giver op nu. Det her er vort daglige brød"

(Brussig 1999/2000: 135/150), 
in dem die Komponenten eines im Deutschen nicht besonders auffallenden Idioms 1:1 übersetzt werden. Die Übersetzung ist stilistisch nicht äquivalent, da das dänische Idiom sehr stark (archaisch) markiert ist. Die rein ikonographische Äquivalenz ist keine Garantie für pragmatische Äquivalenz.

Der Ikonizismus muss aber gar nicht so genau am Ausgangsidiom festhalten um Probleme zu verursachen. Auch in diesem Fall, in dem die Form des dänischen Idioms erheblich geändert worden ist, ist der Ikonizismus im Spiel:

(14.) $[\mathrm{Ich}][\ldots]$ blamiere mich bis auf die Knochen - [Jeg][...] blamerer mig selv helt ind til skindet

(Hein 2001/2002: 184/168)

Die Unnatürlichkeit des Zielsatzes ist vom Zwang zum Ikonographischen verursacht. Die subtile Konvergenz der beiden Ikonographien ist denn auch identifizierbar: In beiden Fällen werden Körperteile verwendet, um die Intensität des Blamiertseins auszudrücken, nämlich jeweils Knochen und skind ('Haut'). Problematisch an der Übersetzung ist ihre kollokationelle Nicht-Typizität, die beim Lesen stutzig macht, was beim deutschen Idiom kaum der Fall ist: Hier ist auf die Knochen einfach ein konventionelles Intensivierungselement von sich blamieren und keine beabsichtigte Metapher. Die dänische Konstruktion fällt also auf, weil sie sehr ungewöhnlich ist. Hätte man das gleiche Typizitätsniveau wie im deutschen Original treffen sollen, hätte man wahrscheinlich auf die Ikonographie verzichten müssen ${ }^{8}$. Eine Google-Recherche zeigt denn auch, dass der typische Kollokator von blamere sig nicht skind ist, sondern variable nicht-ikonographische Intensivierungselemente wie: grundigt, voldsomt, for vildt, i uhфrt grad, massivt, kraftigt, frygteligt.

Der Ikonizismus führt also nicht nur zu fehlender Textäquivalenz, sondern in seinem missverstandenen "Bilderzwang" auch zu Problemen in der Sprachproduktion, indem unnatürliche Texte produziert werden.

Der Ikonizismus ist ein anti-arbitraritätstheoretischer und anti-funktionalistischer Zugang zu Idiomen in der Theorie und vor allem in der Praxis. Er setzt einen mehr oder weniger automatischen Zusammenhang von der Ausdrucks- und der Inhaltsseite voraus, und er legt in Funktionszusammenhängen einen zu großen Wert auf die Ikonographie als ein funktionales Merkmal von Idiomen.

\section{$6 \quad$ Zusammenfassung}

Bei den in diesem Beitrag diskutierten drei Begriffen, Ikonographie, Ikonizität und Ikonizismus, handelt es sich um Kernbegriffe für den wissenschaftlichen Umgang mit dem Teilbereich der Phraseologie, der aus den "bildlichen" Phraseologismen besteht, d. h. den Idiomen im engeren Sinn. Diese Begriffe sind trotz gewisser Gemeinsamkeiten keineswegs synonym, sondern sie füllen jeweils eigene Rollen aus:

\footnotetext{
${ }^{8}$ Was kein Defizit sein muss: Die mit Recht beklagte "Blässe" von Übersetzungen - die man anders bezeichnen sollte, weil "Farbreichtum" ein fragwürdiges und jedenfalls kein wissenschaftlich untersuchbares Merkmal ist ist aber auf einer ganz anderen Ebene zu verorten. Und zwar auf der semantischen, denn Übersetzungen sind in der Tat häufig semantisch allgemeiner (und bei Titeln klischeehafter) als ihre Originale.
} 
Die Ikonographie ist das Potenzial von Idiomen i. e. S., auf der Grundlage einer literalen Lesart des Idioms ein mentales Bild beim Sprecher zu evozieren. Es wird bei der Feststellung von idiomatischer Ikonographie nichts über eine eventuelle kommunikative Relevanz dieses Bildes gesagt. Die Perspektive der Ikonographie ist eine semiotisch-psychologische, keine funktionale. Dies wird oft vergessen, wenn aus der Existenz von Ikonographie operative Schlüsse gezogen werden, etwa für die Übersetzung und die bilinguale Lexikographie. Die Ikonographie ist jedoch für die formale Definition einer phraseologischen Subkategorie entscheidend, der Idiome i. e. $S$.

Die Ikonizität ist ein genuin semiotisches Merkmal einiger Idiome: Wenn Idiome ausdrucksseitig ihren semantischen Inhalt (teilweise) zu widerspiegeln scheinen, kann man davon sprechen, dass sie ikonisch sind. Dabei muss betont werden, dass sie (1) deswegen nicht weniger arbiträr sind - zumindest nach der Arbitraritätsauffassung dieser Arbeit -, dass (2) die Ikonizität relativ ist, und (3) dass der "Inhalt", der eventuell mit der Ikonizität transparent wird, nur einen kleinen Teil des tatsächlichen Inhalts, d. h. sämtlicher Gebrauchseigenschaften des Idioms, ausmacht. Damit kann die Ikonizität bei fein granulierten Idiomanalysen kaum eine wesentliche Rolle spielen, auch deswegen, weil sie interlingual sogar irreführend sein kann.

Das zeigt vielleicht am besten das Phänomen des Ikonizismus, des letzten der hier diskutierten Begriffe der Ikon-Familie. Denn der Ikonizismus, der vor allem in angewandten und interlingualen Kontexten zum Vorschein kommt, ist eine Folge der Ikonographie und der Ikonizität von Idiomen. Er stellt einen anti-arbiträren und anti-funktionalistischen Umgang mit Idiomen dar, der (1) in Bedeutungsanalysen der Ikonographie die Hauptrolle zuerkennt, und nicht den induktiv beobachtbaren abstrakten Gebrauchsregeln des Idioms; der (2) davon ausgeht, dass die Ikonographie prinzipiell einen Einblick in die Bedeutung eines Idioms gewährt; und der (3) in angewandt-interlingualen Kontexten den Ausgangspunkt hat, dass kommunikative Äquivalenz vom Grad der Übereinstimmung der Ausdrucksseite und damit der Ikonographie abhängt. Das Problem des Ikonizismus ist omnipräsent in Theorie und Praxis und er ist für die Generierung vieler Äquivalenzprobleme und die Produktion nichtnatürlicher Texte verantwortlich.

\section{Literaturangaben}

Axelsen, Jens (1992): "En anmeldelse af en anmeldelse". Sprint 2: 1-5.

Bergenholtz, Henning/Tarp, Sven (2002): "Die moderne lexikographische Funktionslehre. Diskussionsbeitrag zu neuen und alten Paradigmen, die Wörterbücher als Gebrauchsgegenstände verstehen". Lexicographica 18: 253-263.

Bolinger, Dwight D. (1968): Aspects of Language. New York etc.

Brussig, Thomas (1999): Am kürzeren Ende der Sonnenallee. Frankfurt a. M.

Brussig, Thomas (2000): I den korte ende af Sonnenallee. Übersetzt von Henrik Andersen. Kopenhagen.

Brøgger, Susanne (1997): Jadekatten. Kopenhagen. 
Brøgger, Susanne (2001): Die Jadekatze. Eine Familiensaga. Übersetzt von Gisela Kosubek. Berlin.

Burger, Harald (1989): "'Bildhaft, übertragen, metaphorisch'. Zur Konfusion um die semantischen Merkmale von Phraseologismen". In Gréciano, Gertrud (ed.): Europhras 88. Phraséologie Contrastive. Actes du Colloque International Klingenthal - Strasbourg 12 16 mai 1988. Strasbourg: 17-29.

Burger, Harald (2003): Phraseologie. Eine Einführung am Beispiel des Deutschen. Berlin.

Burger, Harald (im Druck): "Das phraseologische "Bild" - alte Fragen, neue Antworten?". In: Kržišnik, Erika (ed.): Phraseologie in der Sprachwissenschaft und anderen Disziplinen (Akten der Europhras-Tagung in Strunjan/Slowenien, 19.-22. September 2005).

Dobrovol'skij, Dmitrij (1999a): "Kontrastive Phraseologie in Theorie und im Wörterbuch". In: Baur, Rupprecht S./Chlosta, Christoph/Piirainen, Elisabeth (eds.): Wörter in Bildern Bilder in Wörtern. Beiträge zur Phraseologie und Sprichwortforschung aus dem Westfälischen Arbeitskreis. Baltmannsweiler: 107-122.

Dobrovol'skij, Dmitrij (1999b): "On the Cross-Linguistic Equivalence of Idioms". In: Beedham, Christopher (ed.): "Langue" and "Parole" in Synchronic and Diachronic Perspective. Selected Proceedings of the XXXIst Annual Meeting of the Societas Linguistica Europea, St Andrews, 1998. Amsterdam/Oxford: 203-220.

Dobrovol'skij, Dmitrij (2000): "Idioms in Contrast. A functional view". In: Corpas Pastor, Gloria (ed.): Las lenguas de Europa: Estudios de fraseología y traduccíon. Granada: 367-388.

Dobrovol'skij, Dmitrij (2001): "Zur Motivation in der Idiomatik". In Häcki Buhofer, Annelies/Burger, Harald/Gautier, Laurent (eds.): Phraseologiae Amor. Aspekte europäischer Phraseologie. Festschrift für Gertrud Gréciano zum 60. Geburtstag. Baltmannsweiler: 89-98.

Dobrovol'skij, Dmitrij (2002): "Phraseologismen in kontrastiver Sicht". In: Cruse, Allan D. et al. (eds.) (2002): Lexikologie. Ein internationales Handbuch zur Natur und Struktur von Wörtern und Wortschätzen. 1. Halbband. Berlin/New York: 442-451.

Dobrovol'skij, Dmitrij/Piirainen, Elisabeth (2005): Figurative Language. Amsterdam.

Farø, Ken (2004): "Hvornår går man over åen efter vand? Idiomatiske ækvivalensproblemer i leksikologi og leksikografi". LexicoNordica 11: 85-108.

Farø, Ken (2006): Idiomatizität - Ikonizität - Arbitrarität. Beitrag zu einer funktionalistischen Theorie der Idiomäquivalenz. Kopenhagen: Universität Kopenhagen. [Dissertation].

Farø, Ken (im Druck): "Warum Idiome im mehrfachen Sinn arbiträr sind". In: Kržišnik, Erika (ed.): Phraseologie in der Sprachwissenschaft und anderen Disziplinen (Akten der Europhras-Tagung in Strunjan/Slowenien, 19.-22. September 2005).

Fischer, Andreas (1999): "What, if Anything, is Phonological Iconicity?". In: Nänny, Max/Fischer, Olga (eds.): Form miming meaning. Iconicity in language and literature. Amsterdam/Philadelphia: 123-134.

Häcki Buhofer, Annelies (1989): "Psycholinguistische Aspekte der Bildhaftigkeit von Phraseologismen". In: Gréciano, Gertrud (ed.): Europhras 88. Phraséologie Contrastive. 
Actes du Colloque International Klingenthal - Strasbourg 12 - 16 mai 1988. Strasbourg: $165-175$.

Häcki Buhofer, Annelies (1997): "Der Erwerb von Phraseologismen". In: Wimmer, Rainer/Berens, Franz-Josef (eds.): Wortbildung und Phraseologie. Tübingen: 209-232.

Hallsteinsdóttir, Erla (2001): Das Verstehen idiomatischer Phraseologismen in der Fremdsprache Deutsch. Hamburg.

Hein, Christoph (2001): Willenbrock. Frankfurt a. M.

Hein, Christoph (2002): Willenbrock. Übersetzt von Morten Dyssel Mortensen. Kopenhagen.

Herbermann, Clemens-Peter (1981): Wort, Basis, Lexem und die Grenze zwischen Lexikon und Grammatik. München.

Hockett, Charles F. (1958): A Course in Modern Linguistics. New York.

Holdcroft, David (1991): Saussure: Signs, System, and Arbitrariness. Cambridge etc.

Høeg, Peter (1992): Frøken Smillas fornemmelse for sne. Kopenhagen.

Høeg, Peter (1996): Fräulein Smillas Gespür für Schnee. Übersetzt von Monika Wesemann. Frankfurt a. M.

Ingo, Rune (1990): Från källspråk till målspråk. Introduktion i översättningsvetenskab. Lund. Krohn, Karin (1994): Hand und Fuß. Eine kontrastive Analyse von Phraseologismen im Deutschen und Schwedischen. Göteborg.

Lyons, John (1977): Semantics. Cambridge.

Nänny, Max/Fischer, Olga (eds.) (1999): Form miming meaning. Iconicity in language and literature. Amsterdam/Philadelphia.

Panofsky, Erwin (1978): "Ikonographie und Ikonologie. Eine Einführung in die Kunst der Renaissance". In: Panofsky, Erwin (ed.): Sinn und Deutung in der bildenden Kunst. Köln: 36-67.

Popper, Karl R. (1935/ ${ }^{15}$ 2002): The Logic of Scientific Discovery. New York/London.

Rapoport, Anatol (1975): Semantics. New York.

Saussure, Ferdinand de (1916/32001): Grundfragen der allgemeinen Sprachwissenschaft. Berlin/New York.

Schulze, Ingo (1998): Simple Storys. Berlin.

Tarp, Sven (1995): "Wörterbuchfunktionen. Utopische und realistische Vorschläge für die bilinguale Lexikographie". Germanistische Linguistik 127-128: 17-61.

Whitney, William Dwight (1876): Leben und Wachsthum der Sprache. Leipzig. 